\title{
Proton Affinity of Methyl Peroxynitrate
}

Rose M. Ravelo and Joseph S. Francisco

Department of Chemistry and Department of Earth and Atmospheric Science

Purdue University

West Lafayette, Indiana 47907

\section{Supplemental Tables}

Supplemental Table I: Geometry Optimizations for $\mathrm{CH}_{3} \mathrm{OONO}_{2}$

Supplemental Table II: Optimized Geometries for conformers of protonated $\mathrm{CH}_{3} \mathrm{OONO}_{2}$ (bonds, angles, and dihedral angles)

Supplemental Table III: Total and relative energies for the protonated structures of $\mathrm{CH}_{3} \mathrm{OONO}_{2}$ 
Supplemental Table I. Geometry Optimizations for $\mathrm{CH}_{3} \mathrm{OONO}_{2}{ }^{\mathrm{a}, \mathrm{b}}$

\begin{tabular}{|c|c|c|c|}
\hline Coordinate & B3LYP & QCISD & QCISD (T) \\
\hline $\mathrm{CH}^{\prime}$ & 1.094 & 1.094 & 1.096 \\
\hline $\mathrm{CH"}$ & 1.094 & 1.094 & 1.096 \\
\hline CH'" & 1.093 & 1.094 & 1.095 \\
\hline $\mathrm{CO}^{\prime}$ & 1.434 & 1.438 & 1.441 \\
\hline O'O" & 1.409 & 1.421 & 1.432 \\
\hline $\mathrm{O} " \mathrm{~N}$ & 1.506 & 1.474 & 1.509 \\
\hline NO'" & 1.201 & 1.207 & 1.213 \\
\hline $\mathrm{NO}^{\mathrm{IV}}$ & 1.199 & 1.205 & 1.211 \\
\hline $\mathrm{H}^{\prime} \mathrm{CO}^{\prime}$ & 110.7 & 110.3 & 110.3 \\
\hline H"CO' & 111.1 & 110.7 & 110.7 \\
\hline $\mathrm{H}^{\prime \prime C O}$ & 103.7 & 103.6 & 103.6 \\
\hline CO'O" & 108.2 & 107.2 & 106.9 \\
\hline O'O"N & 109.3 & 108.8 & 108.1 \\
\hline O"NO"' & 110.1 & 110.0 & 109.8 \\
\hline $\mathrm{O}^{\prime} \mathrm{NO}^{\mathrm{IV}}$ & 117.1 & 117.7 & 117.3 \\
\hline H'CO'O" & 57.2 & 57.6 & 57.7 \\
\hline H"CO'O" & -66.5 & -65.9 & -65.8 \\
\hline H"'CO'O" & 175.4 & 176.0 & 176.1 \\
\hline CO'O"N & 94.4: & 93.8 & 92.9 \\
\hline O'O"NO"' & 175.7 & 175.0 & 175.7 \\
\hline $\mathrm{O}^{\prime} \mathrm{O} " \mathrm{NO}^{\mathrm{IV}}$ & -4.4 & -5.5 & -4.7 \\
\hline
\end{tabular}

${ }^{\mathrm{a}}$ Bond distances are in Angstroms and base angles are in degrees.

${ }^{\mathrm{b}}$ Optimized using the 6-31G(d) basis set. 
Supplemental Table II. Optimized Geometries for Conformers of Protonated $\mathrm{CH}_{3} \mathrm{OONO}_{2}$ (Bonds) ${ }^{\mathrm{a}}$

\begin{tabular}{|c|c|c|c|c|c|c|c|c|c|c|c|c|}
\hline & & \multicolumn{11}{|c|}{ Coordinates } \\
\hline Species & Method & $\mathrm{CH}^{\prime}$ & $\mathrm{CH}^{\prime \prime}$ & CH"' & $\mathrm{CO}^{\prime}$ & $\mathrm{O}^{\prime} \mathrm{O}^{\prime \prime}$ & $\mathrm{O} " \mathrm{~N}$ & NO"' & $\mathrm{NO}^{\mathrm{IV}}$ & $\mathrm{O}^{\prime \prime} \mathrm{H}^{\mathrm{IV}}$ & $\mathrm{O}^{\prime} \mathrm{H}^{\mathrm{IV}}$ & $\mathrm{O}^{\prime \prime} \mathrm{H}^{\mathrm{IV}}$ \\
\hline \multirow[t]{3}{*}{$\mathrm{CH}_{3} \mathrm{OONO}_{2}$} & B3LYP/6-31G(d) & 1.094 & 1.094 & 1.093 & 1.434 & 1.409 & 1.506 & 1.201 & 1.199 & & & \\
\hline & QCISD/6-31G(d) & 1.094 & 1.094 & 1.094 & 1.438 & 1.421 & 1.474 & 1.207 & 1.205 & & & \\
\hline & QCISD (T)/6-31G(d) & 1.096 & 1.096 & 1.095 & 1.441 & 1.432 & 1.509 & 1.213 & 1.211 & & & \\
\hline \multirow[t]{3}{*}{$\mathrm{CH}_{3} \mathrm{OOHNO}$} & B3LYP/6-31G(d) & 1.091 & 1.093 & 1.093 & 1.448 & 1.423 & 2.073 & 1.156 & 1.153 & 0.985 & & \\
\hline & QCISD/6-31G(d) & 1.094 & 1.094 & 1.094 & 1.443 & 1.460 & 2.402 & 1.137 & 1.133 & 0.980 & & \\
\hline & QCISD (T)/6-31G(d) & 1.096 & 1.095 & 1.095 & 1.445 & 1.476 & 2.379 & 1.147 & 1.143 & 0.982 & & \\
\hline \multirow[t]{3}{*}{$\mathrm{CH}_{3} \mathrm{OHONO} 2$} & B3LYP/6-31G(d) & 1.087 & 1.091 & 1.089 & 1.509 & 1.442 & 1.717 & 1.170 & 1.173 & & 0.987 & \\
\hline & QCISD/6-31G(d) & 1.088 & 1.092 & 1.090 & 1.513 & 1.453 & 1.658 & 1.178 & 1.181 & & 0.991 & \\
\hline & QCISD (T)/6-31G(d) & 1.089 & 1.093 & 1.091 & 1.514 & 1.468 & 1.711 & 1.185 & 1.188 & & 0.993 & \\
\hline \multirow[t]{3}{*}{$\mathrm{CH}_{3} \mathrm{OONO}_{2} \mathrm{H}$} & B3L YP/6-31G(d) & 1.092 & 1.092 & 1.096 & 1.448 & 1.465 & 1.325 & 1.332 & 1.177 & & & 0.990 \\
\hline & QCISD/6-31G(d) & 1.092 & 1.092 & 1.094 & 1.459 & 1.468 & 1.333 & 1.334 & 1.176 & & & 0.993 \\
\hline & QCISD (T)/6-31G(d) & 1.093 & 1.094 & 1.096 & 1.459 & 1.488 & 1.341 & 1.342 & 1.184 & & & 0.996 \\
\hline
\end{tabular}

${ }^{\mathrm{a} B o n d}$ distances are given in Angstrom. 
Supplemental Table II. Optimized Geometries for Conformers of Protonated $\mathrm{CH}_{3} \mathrm{OONO}_{2}$ (Angles) ${ }^{\mathrm{a}}$

\begin{tabular}{|c|c|c|c|c|c|c|c|c|c|c|c|c|c|}
\hline \multirow[b]{2}{*}{ Species } & \multirow[b]{2}{*}{ Method } & \multicolumn{12}{|c|}{ Coordinates } \\
\hline & & $\mathrm{H}^{\prime} \mathrm{CO}{ }^{\prime}$ & $\mathrm{H}^{\prime \prime C O}$ & H"'CO' & CO'O" & $\mathrm{O}^{\prime} \mathrm{O} " \mathrm{~N}$ & O"NO"' & $\mathrm{O}^{\prime \prime} \mathrm{NO}^{\mathrm{IV}}$ & $\mathrm{O}^{\prime} \mathrm{O}^{\prime \prime} \mathrm{H}^{\mathrm{IV}}$ & $\mathrm{H}^{\mathrm{IV}} \mathrm{O} " \mathrm{~N}$ & $\mathrm{CO}^{\prime} \mathrm{H}^{\mathrm{IV}}$ & $\mathrm{H}^{\mathrm{IV}} \mathrm{O}^{\prime} \mathrm{O}^{\prime \prime}$ & $\mathrm{NO}^{\prime \prime} \mathrm{H}^{\mathrm{IV}}$ \\
\hline \multirow{3}{*}{$\mathrm{CH}_{3} \mathrm{OONO}_{2}$} & B3LYP/6-31G(d) & 110.7 & 111.1 & 103.7 & 108.2 & 109.3 & 110.1 & 117.1 & & & & & \\
\hline & QCISD/6-31G(d) & 110.3 & 110.7 & 103.6 & 107.2 & 108.8 & 110.0 & 117.7 & & & & & \\
\hline & QCISD (T)/6-31G(d) & 110.3 & 110.7 & 103.6 & 106.9 & 108.1 & 109.8 & 117.3 & & & & & \\
\hline \multirow[t]{3}{*}{$\mathrm{CH}_{3} \mathrm{OOHNO}{ }_{2}$} & B3L YP/6-31G(d) & 110.0 & 110.7 & 102.2 & 107.8 & 102.4 & 106.2 & 100.5 & 101.6 & 107.7 & & & \\
\hline & QCISD/6-31G(d) & 110.4 & 110.6 & 103.5 & 107.0 & 95.7 & 95.4 & 89.9 & 101.7 & 129.3 & & & \\
\hline & QCISD (T)/6-31G(d) & 110.4 & 110.7 & 103.3 & 106.6 & 95.3 & 95.9 & 90.4 & 101.0 & 128.2 & & & \\
\hline \multirow[t]{3}{*}{$\mathrm{CH}_{3} \mathrm{OHONO}_{2}$} & B3LYP/6-31G(d) & 104.3 & 103.9 & 108.0 & 111.4 & 107.2 & 104.7 & 112.5 & & & 112.6 & 104.2 & \\
\hline & QCISD/6-31G(d) & 103.9 & 103.8 & 107.8 & 109.7 & 106.5 & 104.9 & 113.3 & & & 112.2 & 103.3 & \\
\hline & QCISD (T)/6-31G(d) & 103.9 & 103.8 & 107.9 & 109.3 & 105.7 & 104.7 & 112.8 & & & 111.8 & 102.7 & \\
\hline \multirow[t]{3}{*}{$\mathrm{CH}_{3} \mathrm{OONO}_{2} \mathrm{H}$} & B3LYP/6-31G(d) & 110.8 & 110.8 & 99.9 & 105.3 & 106.6 & 106.8 & 126.6 & & & & & 106.1 \\
\hline & QCISD/6-31G(d) & 110.1 & 110.1 & 100.6 & 104.0 & 105.4 & 106.5 & 126.7 & & & & & 106.1 \\
\hline & QCISD (T)/6-31G(d) & 110.2 & 110.4 & 100.6 & 103.9 & 104.9 & 106.1 & 126.9 & & & & & 105.9 \\
\hline
\end{tabular}

${ }^{\mathrm{a} B o n d}$ angles are given in degrees. 
Supplemental Table II. Optimized Geometries for Conformers of Protonated $\mathrm{CH}_{3} \mathrm{OONO}_{2}$ (Dihedral Angles) ${ }^{\mathrm{a}}$

\begin{tabular}{|c|c|c|c|c|c|c|c|c|c|c|c|c|c|c|c|}
\hline \multirow[b]{2}{*}{ Species } & \multirow[b]{2}{*}{ Method } & \multirow[b]{2}{*}{ H'CO'O" } & \multirow[b]{2}{*}{ H"CO'O" } & \multicolumn{12}{|c|}{ Coordinates } \\
\hline & & & & H"'CO'O" & $\mathrm{CO}^{\prime} \mathrm{O} " \mathrm{~N}$ & O'O"N"' & $\mathrm{O}^{\prime} \mathrm{O} " \mathrm{NO}^{\mathrm{IV}}$ & $\mathrm{CO}^{\prime} \mathrm{O}^{\prime \prime} \mathrm{H}^{\mathrm{IV}}$ & $\mathrm{H}^{\mathrm{IV}} \mathrm{O}^{\prime \prime} \mathrm{NO}^{\prime \prime}$ & $\mathrm{H}^{\mathrm{IV}} \mathrm{O}^{\prime \prime} \mathrm{NO}^{\mathrm{IV}}$ & $\mathrm{H}^{\prime} \mathrm{CO}^{\prime} \mathrm{H}^{\mathrm{IV}}$ & $\mathrm{H}^{\prime \prime} \mathrm{CO}^{\prime} \mathrm{H}^{\mathrm{IV}}$ & $\mathrm{H}^{\prime \prime} \mathrm{CO}^{\prime} \mathrm{H}^{\mathrm{IV}}$ & $\mathrm{H}^{\mathrm{IV}} \mathrm{O}^{\prime} \mathrm{O}^{\prime \prime} \mathrm{N}$ & O"NO"'H ${ }^{\mathrm{IV}}$ \\
\hline \multirow{3}{*}{$\mathrm{CH}_{3} \mathrm{OONO} \mathrm{O}_{2}$} & B3LYP/6-31G(d) & 57.2 & $\begin{array}{l}-66.5 \\
\end{array}$ & 175.4 & 94.4 & 175.7 & $\begin{array}{l}-4.4 \\
\end{array}$ & & & & & & & & \\
\hline & QCISD/6-31G(d) & 57.6 & -65.9 & 176.0 & 93.8 & 175.0 & $-5.5^{`}$ & & & & & & & & \\
\hline & QCISD (T)/6-31G(d) & 57.7 & -65.8 & 176.1 & 92.9 & 175.7 & -4.7 & & & & & & & & \\
\hline \multirow[t]{3}{*}{$\mathrm{CH}_{3} \mathrm{OOHNO} \mathrm{H}_{2}$} & B3LYP/6-31G(d) & 63.4 & -60.6 & -178.5 & 109.5 & 173.7 & -7.8 & -139.2 & 67.1 & -114.5 & & & & & \\
\hline & QCISD/6-31G(d) & 64.2 & -58.9 & -177.7 & 132.6 & 161.8 & -18.6 & -95.3 & 51.7 & -128.8 & & & & & \\
\hline & QCISD (T)/6-31G(d) & 64.1 & -59.2 & -177.9 & 132.2 & 164.1 & -16.2 & 132.2 & 55.6 & -124.7 & & & & & \\
\hline \multirow[t]{3}{*}{$\mathrm{CH}_{3} \mathrm{OHONO}{ }_{2}$} & B3LYP/6-31G(d) & -66.0 & 176.1 & 54.9 & 121.4 & 179.4 & -0.7 & & & & 177.4 & 59.5 & -61.7 & -117.0 & \\
\hline & QCISD/6-31G(d) & -66.5 & 175.5 & 54.2 & 120.9 & 178.6 & -1.7 & & & & 179.4 & 61.4 & -59.9 & -119.4 & \\
\hline & QCISD (T)/6-31G(d) & -66.2 & 175.7 & 54.4 & 121.3 & 178.7 & -1.6 & & & & -179.3 & 62.6 & -58.7 & -119.8 & \\
\hline \multirow[t]{3}{*}{$\mathrm{CH}_{3} \mathrm{OON} 0_{2} \mathrm{H}$} & B3LYP/6-31G(d) & 63.4 & -63.3 & -180.0 & -179.8 & -180.0 & 0.03 & & & & & & & & -180.0 \\
\hline & QCISD/6-31G(d) & 62.7 & -62.7 & 180.0 & -180.0 & -180.0 & 0.004 & & & & & & & & -180.0 \\
\hline & QCISD (T)/6-31G(d) & 64.0 & -61.5 & -179.0 & -177.7 & -179.4 & 0.6 & & & & & & & & -179.3 \\
\hline
\end{tabular}

${ }^{\mathrm{a} B o n d}$ angles are given in degrees. 
Supplemental Table III. Total and Relative Energies ${ }^{\mathrm{a}}$ for the Protonated Structures of $\mathrm{CH}_{3} \mathrm{OONO}_{2}$

\begin{tabular}{ccccccc}
\hline & \multicolumn{3}{c}{ Total Energies (Hartrees) } & \multicolumn{3}{c}{ Relative Energies (kcal/mol) } \\
\cline { 2 - 7 } \multicolumn{1}{c}{ Species } & B3LYP & QCISD & QCISD (T) & B3LYP & QCISD & QCISD (T) \\
\hline $\mathrm{CH}_{3} \mathrm{OONO}_{2}$ & -395.32201 & -394.27737 & -394.31732 & 0.0 & 0.0 & 0.0 \\
$\mathrm{CH}_{3} \mathrm{OOHNO}_{2}$ & -395.60887 & -394.56388 & -394.60461 & 173.7 & 173.5 & 174.0 \\
$\mathrm{CH}_{3} \mathrm{OHONO}_{2}$ & -395.59418 & -394.54502 & -394.58829 & 163.7 & 160.9 & 162.9 \\
$\mathrm{CH}_{3} \mathrm{OONO}_{2} \mathrm{H}$ & -395.59503 & -394.55009 & -394.58762 & 163.9 & 163.7 & 162.2 \\
\hline
\end{tabular}

${ }^{\mathrm{a}}$ Species in red is the lowest energy structure for protonated $\mathrm{CH}_{3} \mathrm{OONO}_{2}$.

${ }^{\mathrm{b}}$ All energies are determined from optimized structure, using the 6-31G(d) basis set. 